\title{
EHMTI-0227. Therapeutic effectiveness of sphenopalatine ganglion (SPG) stimulation for cluster headache - pathway registry study interim results at 6 months
}

\author{
TP Jürgens ${ }^{1 *}$, R Jensen ${ }^{2}$, H Kaube ${ }^{3}$, A Böger ${ }^{4}$, C Gaull$^{5}$, A Goodman ${ }^{6}$, A Caparso ${ }^{6}$, A May ${ }^{1}$ \\ From 4th European Headache and Migraine Trust International Congress: EHMTIC 2014 \\ Copenhagen, Denmark. 18-21 September 2014
}

\section{Background}

The Pathway Registry is an open label registry of SPG stimulation therapy for cluster headache treatment. In a previous randomized, double-blind, multi-center study (Pathway $\mathrm{CH}-1$ study) $68 \%$ of patients experienced clinically significant improvements.

\section{Aim}

The aim of this interim analysis is to evaluate acute and/or preventive therapeutic effectiveness at 6 months following insertion of an SPG neurostimulator.

\section{Method}

Therapeutic effectiveness (acute pain response following SPG stimulation and/or attack frequency reduction) was analyzed during the first six months following SPG neurostimulator insertion. Acute pain responders achieved relief from moderate or greater pain, or freedom from mild pain in $\geq 50 \%$ of analyzable attacks (with completed diary questions). Frequency responders achieved $\geq 50 \%$ attack frequency reduction at the six month study visit (evaluated over the previous four weeks), versus the 4 week baseline period.

\section{Results}

49 patients have been enrolled, 18 completed follow-up through six months[RJ1] (189 days post-insertion, range 149-238). Average baseline attack frequency was 28.4 attacks/week (range 0-70), and the average attack frequency at 6 months was 17.3 attacks/week (range 0-70), a $40 \%$ reduction. $67 \%(12 / 18)$ were responders. Of the 12 responders, $67 \%(\mathrm{~N}=8)$ were acute responders, treating $86 \%$ of their attacks effectively $(\mathrm{N}=546) .75 \%(\mathrm{~N}=9)$ were frequency responders; frequency reduced by $90 \%$ (from 22.3 at baseline to 2.1 (range 0-8) attacks/week). 5 patients were both acute and frequency responders.

\section{Conclusion}

Interim data from a registry of cluster headache patients continues to demonstrate the effectiveness of SPG stimulation therapy.

\section{Authors' details}

${ }^{1}$ Headache Clinic Dept. of Systems Neuroscience, Medical Center HamburgEppendorf, Hamburg, Germany. ${ }^{2}$ Danish Headache Center, Glostrup Hospital University of Copenhagen, Copenhagen, Denmark. ${ }^{3}$ Neurologie und Kopfschmerzzentrum Muenchner Freiheit, Neurologie und Kopfschmerzzentrum Muenchner Freiheit, Munich, Germany. ${ }^{4}$ Schmerzzentrum, Rotes Kreuz Krankenhaus, Kassel, Germany. ${ }^{5}$ Migräne und Kopfschmerzklinik, Migräne und Kopfschmerzklinik, Königstein, Germany. ${ }^{6}$ Autonomic Technologies Inc., Autonomic Technologies Inc., Redwood City California, USA.

Published: 18 September 2014

doi:10.1186/1129-2377-15-S1-C26

Cite this article as: Jürgens et al.: EHMTI-0227. Therapeutic effectiveness of sphenopalatine ganglion (SPG) stimulation for cluster headache pathway registry study interim results at 6 months. The Journal of Headache and Pain 2014 15(Suppl 1):C26. 